\title{
Soft X-ray Microscopy: History, Status, and Future
}

\author{
Harald Ade ${ }^{1}$
}

1. Dept. of Physics and Organic and Carbon Electronics Laboratory (ORaCEL), North Carolina State University, Raleigh, NC 27695

Photon-in, photon-out X-ray microscopy traces its roots all the way back to Nobel Laureate Wilhelm Röntgen, who has as one of the first applications used X-rays in 1895-1897 to image the internal structures of various objects, one of the most famous being the hand of his wife (see Figure 1). Since then, X-ray microscopy has yielded many unique developments and blossomed into many specialized techniques.

One major and important subcategory of X-ray microscopy is soft X-ray microscopy based on zone plate optics, pioneered in the 1970s and 1980s and perfected over the years. The interaction of soft Xrays with matter provides unique advantages, such as low radiation dose and sensitivity to chemical composition and bonding via the spectral signature close to elemental absorption edges [1]. These spectroscopic capabilities were particularly useful, as soft X-rays in the energy range of 200 to $900 \mathrm{eV}$ covered many important $\mathrm{K}$ edges, such as a carbon, oxygen and nitrogen, as well as L,M edges of transition metals and their various compounds. This leads to applications in a wide range of fields ranging from magnetism, polymer science, biology, chemistry, catalysis, to meteoritics, environmental studies and mineralization.

Unfortunately, these methods and applications require high brightness sources, very special, expensive and almost one-of-kind optics, and exquisite energy tunability. For these reasons, use and development of soft X-ray microscopy remains largely chained to synchrotron radiation facilities. There, the user base has steadily grown and existing instruments are highly productive and consistently oversubscribed. As the complexity of the applications has increased, for example in battery research, the need for improved spatial resolution, sensitivity, and sample environment control has pushed continual improvements in instrument capabilities. Furthermore, substantial future investments into synchrotron radiation facilities upgrades are often based on pushing back the frontiers of spatial resolution, energy resolution, and accessible time scales.

We will trace some of the key developments of soft X-ray microscopy, illustrate the power of the method by presenting representative applications, and make links to soft X-ray scattering methods that exploit similar spectroscopic principles, but provide only statistical information about real space relations due to the sampling of data in reciprocal space [2]. We also discuss the extension of scanning transmission X-ray microscopy to ptychography, a method that records reciprocal space information to recover the phase of the field lost in an amplitude measurement and allows reconstruction of real space information beyond the spot size of the probe beam $[3,4]$. X-ray ptychography has now reached spatial resolution below $10 \mathrm{~nm}$, but retains a lot of the analytical capabilities derived from soft X-ray spectroscopy.

References:

[1] H. Ade et al, Science (1990). 
[2] H. Ade and A.P. Hitchcock, Polymer 49, 643-675 (2008)

[3] P. Thibault et al, Science 321, 379-382 (2008)

[4] D. A. Shapiro et al, Nature Photonics 8, 765-769 (2014)

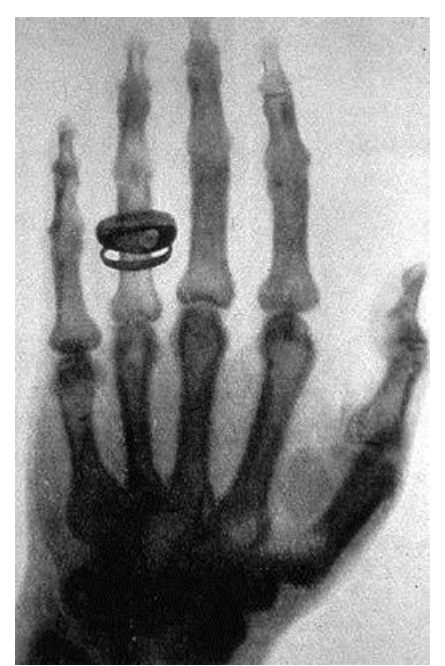

Figure 1. One of the first medical X-rays by Wilhelm Röntgen of the hand of his wife Anna Bertha Ludwig

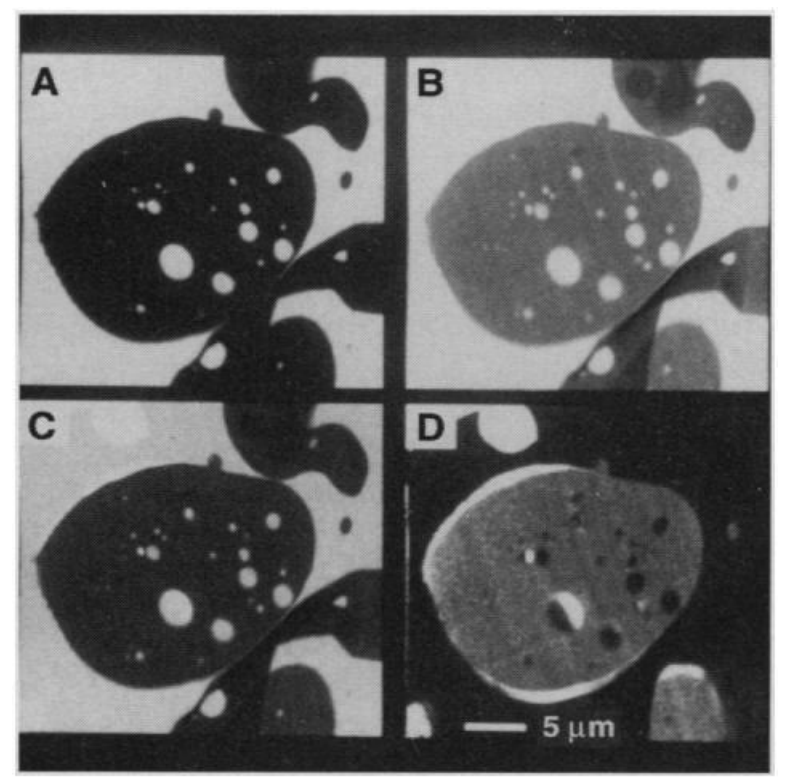

Figure 2. First use of near edge $\mathrm{x}$-ray absorption fine-structure spectroscopy in a transmission $\mathrm{x}$-ray microscope [1]. Images of a 0.5 -m-thick section of a polypropylene:styrene-acrylonitrile copolymer blend at the photon energies of(A) $285.5 \mathrm{eV}$, (B) $286.2 \mathrm{eV}$, (C) $286.8 \mathrm{eV}$, and (D) $287.9 \mathrm{eV}$, respectively. The contrast arises from differences in the near-edge absorption cross section of the different domains as. Conceptually, this is very similar to EELS imaging, but offers lower radiation dose and higher spectral resolution. 